\title{
Diverging Playing Positions do not Lead to Differences in YBT-UQ Performance in Youth Handball Players
}

\section{() (1) $(8) \ominus$}

\author{
Authors \\ Julian Bauer ${ }^{\mathbb{D}}$, Gerrit Schwiertz, Thomas Muehlbauer
}

\begin{abstract}
Affiliation
Division of Movement and Training Sciences/Biomechanics of Sport, University of Duisburg-Essen, Essen, Germany
\end{abstract}

Key words

young players, overhead athletes, functional testing, shoulder mobility/stability

$\begin{array}{ll}\text { received } & 13.04 .2021 \\ \text { revised } & 15.07 .2021 \\ \text { accepted } & 25.07 .2021\end{array}$

\section{Bibliography}

Sports Medicine International Open 2021; 5: E99-E103

DOI 10.1055/a-1647-7174

ISSN 2367-1890

(C) 2021. The Author(s).

This is an open access article published by Thieme under the terms of the Creative Commons Attribution-NonDerivative-NonCommercial-License, permitting copying and reproduction so long as the original work is given appropriate credit. Contents may not be used for commercial purposes, or adapted, remixed, transformed or built upon. (https://creativecommons. org/licenses/by-nc-nd/4.0/)

Georg Thieme Verlag KG, Rüdigerstraße 14,

70469 Stuttgart, Germany

\section{Correspondence}

Julian Bauer, PhD

Division of Movement and Training Sciences/Biomechanics

of Sport

University of Duisburg-Essen

Gladbecker Straße 182

45141 Essen

Germany

Tel.: +49/201/183 7325

julian.bauer@uni-due.de

\begin{abstract}
Handball playing positions can be differentiated between first line players who position themselves near the 6-m line and second line players who typically play outside the 9 -m space. Handball is characterized by a high number of throwing actions that cause adaptations in the throwing shoulder. The objective of this cross-sectional study was to assess whether the specific physiological positional demands in handball lead to functional performance differences between the playing positions ( $N=196$; goalkeepers: $n=25$; backcourt: $n=99$; pivots: $n=21$; winger: $n=51$ ) in terms of shoulder mobility and stability in any reach direction as assessed through the Upper Quarter Y Balance Test (YBT-UQ). Contrary to our hypothesis, the results did not show significant differences between the playing positions in shoulder mobility and stability in youth handball players, irrespective of reach arm and reach direction. The obtained effect sizes $\left(\eta_{p}^{2}\right)$ were solely small and ranged between 0.01 and 0.03 . The adaptations following the demands of the diverging playing positions do not lead to significant differences in shoulder mobility and stability on the basis of the YBT-UQ. The overall training load of youth handball players may not be sufficient to lead to significant position-specific differences in shoulder mobility and stability.
\end{abstract}

\section{Introduction}

In team sports, different positional demands in terms of tactics, physical characteristics and technique are reported [1]. One of these team sports is handball, which is a fast-paced intermittent high-intensity Olympic contact sport [2]. Handball playing positions in offense can be divided into first line players who are posi- tioned near the 6-m line and the second line players who are typically positioned outside the 9 -m space. Pivots and wingers are therefore considered as first line players, whereas backcourt players, i. e., back left and back right as well as the center, are second line players. Handball is characterized by a high number of throwing and passing actions nearly exclusively with the throwing arm 
that cause specific adaptations [3]. While throwing, basically two different arm positions can be differentiated: (a) the overhead throw, which is a technique characterized by moving the elbow above shoulder height following the wind-up and acceleration phase. In contrast, (b) the side-arm throw is a technique during which the arm, elbow, and hand are below or maximally at shoulder height during the acceleration and deceleration phase as well as the follow-through phase [4]. Both types of throws differ considerably in terms of kinetics and the relevant kinematic body angles and angular velocities [4]. Higher values of shoulder flexion and lower values of shoulder abduction at ball release have been reported when using the side-arm throw compared with the overhead throw [4]. Although the proximal-to-distal sequence is independent of the arm position, significantly fewer throws are executed as side-arm throws, which also display a reduced ball release speed and velocity [4].

In playing situations, the first line players seldom have to throw over a defender owing to their position near the goal. Therefore, side-arm throws with a long arm are especially present in throws of the wingers together with curved jumps to improve the angles from the outer positions mostly with no opposition besides the goalkeeper in front of them [5]. These throwing necessities typically lead to the first line players executing throws with less throwing velocity but higher demands in terms of accuracy $[6,7]$. The second line players often have to throw over defenders from wider distances [5]. Consequently, backcourt players mostly use overhead throws with a long arm being extended more into a horizontal direction to be able to throw over defenders in front of them who try to block. Additionally, backcourt players typically position themselves within the central zone in which body contact and 1 against 1 situations are more frequently displayed than on the wings [8-10]. Based on these differences, it might be possible that the functional adaptation in terms of shoulder mobility and stability differs between playing positions, especially between the first and second line players. Goalkeepers do represent a distinctive playing position as they normally only play in defense [9]. Goalkeepers need to be rather tall while remaining flexible enough to save throws from different angles with their whole body [11]. It could therefore be assumed, that goalkeepers display higher mobility and stability in the shoulder girdle due to their increased demands in terms of flexibility when saving throws. However, the functional adaptation and influence of throws executed by goalkeepers themselves is less likely, as they mostly perform short passes and considerably fewer throws with much lower velocities.

Functional adaptations may be helpful for the specific demands of the individual playing positions (e. g., throwing with a long arm resembling the medial reach direction to improve the angles for the wingers and throwing over a block resembling the superolateral reach direction for the backcourt players) in handball. Therefore, identifying positional differences that may be advantageous in terms of reach directions and distances for any playing position may allow developing position-specific programs aiming to improve shoulder mobility and stability in youth handball players. The present study is the first one to assess the functional adaptations in shoulder mobility and stability based on the different playing positions in youth handball players.
The Upper Quarter Y Balance Test (YBT-UQ) is a closed kinetic chain test [12] with the potential to assess shoulder mobility and stability in interaction with the trunk [13]. The YBT-UQ was used as handball is played mainly unilaterally with a rather heavy ball and the high number of throws and passes have been reported to lead to significant asymmetries in different age groups and reach directions in adolescent handball players [14] as well as adult water polo players [15]. Therefore, the objective of the cross-sectional study was to assess whether the specific physiological positional demands in handball lead to functional performance differences between the playing positions in terms of shoulder mobility and stability in any reach direction as assessed through the YBT-UQ. We hypothesized that significant differences between the playing positions due to their diverging demands were present in youth handball players.

\section{Methods}

\section{Participants}

An a priori power analysis using $G$ * Power [16] with the following input parameters was performed: effect size $(f=0.25)$, type I error $(\alpha=0.05)$, type II error $(1-\beta=0.80)$, and number of groups $(n=4)$. The analysis revealed that a total sample size of $N=180$ participants would be sufficient to find significant group differences. The characteristics of the study population by playing position are summarized in > Table 1. All players are members of regional youth teams with a training frequency in their respective clubs of 3-4 training sessions per week. The players and their parents or legal guardians were informed about the study procedure, testing protocol, and possible risks. Written consent of all players and informed consent of the parents or legal guardians was obtained before the testing. The cross-sectional study was conducted according to the Ethical Standards in Sport and Exercise Science Research [17]. The study was approved by the human ethics committee of the Faculty of Educational Sciences, University of Duisburg-Essen (TM_24.03.20) and it was executed in line with the Declaration of Helsinki.

\section{Testing procedures}

The testing was carried out on four different measurement dates with four different testing groups. Every participant executed a standardized warm-up including five minutes of submaximal running and a mobility routine prior to the testing. Anthropometric and YBT-UQ assessments were executed in a random order with identical graduated testing personnel being present at all testing sessions. Standardized verbal instruction was given prior to the execution of the YBT-UQ.

\section{Assessment of anthropometric characteristics}

From the seventh cervical spinous process to the distal tip of the middle finger, upper limb length was measured with the shoulder in $90^{\circ}$ abduction [13]. A Seca clara 803 digital scale was used to assess body mass $(\mathrm{kg})$. Standing body height $(\mathrm{cm})$ without shoes was assessed with a Seca linear measure scale. Players stated their training experience in years and their dominant playing position together with their dominant and throwing arm. 
- Table 1 Characteristics of the study participants $(N=196)$ by player position.

\begin{tabular}{|c|c|c|c|c|c|}
\hline Characteristic & Goalkeeper $(n=25)$ & Backcourt $(n=99)$ & Pivots $(n=21)$ & Wingers $(n=51)$ & $p-\mid \eta_{p}^{2}$-value \\
\hline $\operatorname{Sex}(f / m)$ & $9 / 16$ & $46 / 53$ & $9 / 12$ & $22 / 29$ & - \\
\hline Age (yrs) & $14.8 \pm 1.7$ & $15.2 \pm 1.6$ & $15.7 \pm 1.4$ & $15.4 \pm 1.5$ & $0.27(0.02)$ \\
\hline Body height $(\mathrm{cm})$ & $174.9 \pm 10.2$ & $176.8 \pm 9.3$ & $179.1 \pm 9.5$ & $171.8 \pm 7.9$ & $<0.01(0.07)$ \\
\hline Body mass $(\mathrm{kg})$ & $72.4 \pm 15.3$ & $71.0 \pm 12.4$ & $77.3 \pm 13.0$ & $62.5 \pm 9.8$ & $<0.01(0.13)$ \\
\hline BMI $\left(\mathrm{kg} / \mathrm{m}^{2}\right)$ & $22.5 \pm 5.8$ & $22.6 \pm 3.0$ & $24.1 \pm 2.9$ & $21.2 \pm 3.2$ & $<0.05(0.06)$ \\
\hline Left arm length $(\mathrm{cm})$ & $88.9 \pm 6.6$ & $89.1 \pm 5.5$ & $90.6 \pm 4.9$ & $86.5 \pm 4.7$ & $<0.05(0.06)$ \\
\hline Right arm length $(\mathrm{cm})$ & $89.3 \pm 7.3$ & $89.6 \pm 5.1$ & $91.2 \pm 4.8$ & $86.8 \pm 5.0$ & $<0.01(0.07)$ \\
\hline Arm dominance $(I / r)$ & $1 / 24$ & $7 / 92$ & $2 / 19$ & $7 / 44$ & - \\
\hline Throwing arm (I/r) & $0 / 25$ & $6 / 93$ & $0 / 21$ & $9 / 42$ & - \\
\hline Training experience (yrs) & $8.1 \pm 3.3$ & $8.0 \pm 2.9$ & $8.3 \pm 2.7$ & $8.6 \pm 3.0$ & $0.81(0.01)$ \\
\hline
\end{tabular}

Assessment of Upper Quarter Y Balance Test performance The Y Balance Test Kit (Move2Perform, Evansville, IN, USA) was used to assess the three reach directions: medial (MD), inferolateral (IL), and superolateral (SL). Each participant performed three submaximal practice trials followed by three maximal data-collection trials per arm and reach direction (sequence: $\mathrm{MD}$ then IL then $\mathrm{SL}$ ). One of the testers gave a standardized verbal instruction prior to the execution. The left arm had to be placed at the center of the junction to reach out to the furthest point of each reach direction with the right arm. This protocol was then replicated for the other body side. Breaks of five minutes and one minute were given between the right and the left arm reach trials and single reach trials, respectively. Trials were invalid if three-point contact was lost or the participant touched the floor with the mobile arm or hand [12]. The best value (i. e., maximal reach distance) was determined to the nearest $0.5 \mathrm{~cm}$ for every reach direction being normalized for arm length (AL) and an additional normalized composite score (CS) as the mean of the averaged maximal distances in all reach directions was calculated. Using similar procedures, Schwiertz et al. [18] reported sufficient test-retest reliability $\left(\mathrm{ICC}_{3,1}: 0.47-0.83\right.$, SEM: $3.4-$ $7.6 \%, \mathrm{MDC}_{95} \%$ : 9.5-21.1\%) for these measures in healthy youth ( 15 years).

\section{Statistical analysis}

Mean values and standard deviations were calculated and YBT-UQ performance differences between groups of player position were investigated by using an analysis of variance (ANOVA). Normal distribution was examined and confirmed $(p>0.05)$ using the Shapiro-Wilk test. Furthermore, effect size $\left(\eta_{\mathrm{p}}{ }^{2}\right)$ was calculated and classified as small $\left(0.02 \leq \eta_{\mathrm{p}}{ }^{2} \leq 0.12\right)$, medium $\left(0.13 \leq \eta_{\mathrm{p}}{ }^{2} \leq 0.25\right)$, and large $\left(\eta_{\mathrm{p}}^{2} \geq 0.26\right)$. The level of statistical significance was set at $p<0.05$. All statistical analyses were made with the Statistical Package for Social Sciences, version 27.0 (IBM Corp., Armonk, NY, USA).

\section{Results}

The mean values and standard deviations for the normalized YBTUQ performance by reach arm and player position are shown in - Table 2. Irrespective of reach arm and reach direction, the ANOVA revealed no significant performance differences between player positions. In addition, the obtained effect sizes were solely small and ranged between 0.01 and 0.03 .

\section{Discussion}

Contrary to our expectation, the results did not show significant differences between the playing positions in shoulder mobility and stability as assessed through the YBT-UQ in youth handball players. Additionally, anthropometric differences that are well documented in senior players [5] were partially present between the different playing positions in the present sample. More specifically, significant anthropometric differences between playing positions were present with the pivots being taller and heavier than the wingers ( $\triangleright$ Table $\mathbf{1})$.

Owing to the lack of studies on position-specific performance differences in shoulder mobility and stability, we compared our findings with results originating from a similar approach (i. e., differences in lower-extremity mobility and stability according to playing positions). For instance and contrary to our findings, Patel and Choudhary [19] were able to detect significant differences in balance performance as assessed through the long nose test between the playing positions of handball players in favor of right wingers when compared with center players $(p=0.001)$, goalkeepers $(p<0.001)$, back left $(p=0.003)$, and back right $(p<0.001)$ as well as pivots $(p<0.001)$. Further, Brumitt et al. [20] reported significant differences in the Lower Quarter Y-Balance Test (YBT-LQ) between volleyball players with different playing positions, with liberos, defensive specialists, and steers taken together displaying significantly better results (posterolateral reach direction: $106.1 \pm 10.7 \%$ leg length, $p=0.001$; CS: $106.9 \pm 12.8 \%$ leg length, $p<0.001)$ than the outside hitters, middle blockers, and opposite right side hitters (posterolateral reach direction: $99.8 \pm 10.6 \%$ leg length; CS: $94.8 \pm 10.8 \%$ leg length) when the right lower extremity was tested. Also for the left lower extremity, significantly (posterolateral reach direction: $p=0.002 ; \mathrm{CS}: p<0.001$ ) better results were reported for the liberos, defensive specialists, and steers taken together (posterolateral reach direction: $105.8 \pm 9.1 \%$ leg length; CS: $1070.0 \pm 11.4 \%$ leg length) than for the outside hitters, middle blockers, and opposite right hitters taken together (posterolateral reach direction: $100.1 \pm 10.8 \%$ leg length; CS: $95.6 \pm 10.4 \%$ leg 
- Table 2 Comparison of Upper Quarter Y Balance Test performance by player position.

\begin{tabular}{|c|c|c|c|c|c|}
\hline Outcome & Goalkeeper $(n=25)$ & Back $(n=99)$ & Pivot $(n=21)$ & Winger $(n=51)$ & $p$ - $\mid \eta_{\mathrm{p}}{ }^{2}$-value \\
\hline \multicolumn{6}{|c|}{ Right arm reach } \\
\hline $\mathrm{MD}(\% \mathrm{AL})$ & $98.5 \pm 18.2$ & $106.2 \pm 14.6$ & $107.2 \pm 18.8$ & $106.9 \pm 13.8$ & $0.12(0.03)$ \\
\hline $\mathrm{IL}(\% \mathrm{AL})$ & $102.4 \pm 16.8$ & $103.8 \pm 15.9$ & $105.5 \pm 17.3$ & $104.7 \pm 13.8$ & $0.90(0.01)$ \\
\hline $\mathrm{SL}(\% \mathrm{AL})$ & $83.0 \pm 11.5$ & $84.3 \pm 11.5$ & $84.3 \pm 13.8$ & $85.2 \pm 11.2$ & $0.89(0.01)$ \\
\hline $\mathrm{CS}(\% \mathrm{AL})$ & $94.6 \pm 9.0$ & $98.1 \pm 8.7$ & $99.0 \pm 8.3$ & $99.0 \pm 8.7$ & $0.20(0.02)$ \\
\hline \multicolumn{6}{|l|}{ Left arm reach } \\
\hline $\mathrm{MD}(\% \mathrm{AL})$ & $97.7 \pm 19.0$ & $104.7 \pm 13.6$ & $105.3 \pm 16.5$ & $105.6 \pm 12.9$ & $0.13(0.03)$ \\
\hline $\mathrm{IL}(\% \mathrm{AL})$ & $104.8 \pm 16.0$ & $102.1 \pm 16.6$ & $103.4 \pm 16.5$ & $103.8 \pm 14.4$ & $0.87(0.01)$ \\
\hline $\mathrm{SL}(\% \mathrm{AL})$ & $80.2 \pm 13.7$ & $82.6 \pm 11.4$ & $82.3 \pm 9.7$ & $84.3 \pm 12.7$ & $0.55(0.01)$ \\
\hline $\mathrm{CS}(\% \mathrm{AL})$ & $94.2 \pm 9.7$ & $96.4 \pm 9.2$ & $97.0 \pm 8.2$ & $97.9 \pm 9.2$ & $0.42(0.01)$ \\
\hline
\end{tabular}

Data are mean \pm standard deviation. $\mathrm{AL}=$ arm length; $\mathrm{CS}=$ composite score; $\mathrm{IL}=$ inferolateral; $\mathrm{MD}=$ medial; $\mathrm{SL}=$ superolateral.

length). In baseball, Ryu et al. [21] were able to detect significant differences between the pitchers ( $107.7 \pm 6.8 \%$ leg length) and infielders $(113.7 \pm 6.8 \%$ leg length) in mean right posteromedial normalized reach distances $(p=0.028)$ and the CS (pitchers: $92.3 \pm 4.3 \%$ leg length; infielders: $96.6 \pm 6.2 \%$ leg length $)(p=0.048)$ executing the YBT-LQ. Studies assessing side differences between the upper extremities in trained high school baseball/softball players [22] as well as volleyball and basketball players [23] did not report significant differences following the unilateral execution of these sports although these studies did not subdivide the athletes into their playing positions.

What are likely explanations for our finding that YBT-UQ performance did not differ according to player position? First, the anthropometric differences between the playing positions are rather low except for pivots compared to wingers. Taller players who more often play as second line players may more often use the overhead throw due to the often present opposition in front of them that tries to block, whereas the smaller players who more often play as first line players with no opposition in front of them often use the side arm throw to improve angles. However, these anthropometric differences and the possible subsequent adaptations were not or only partially present in the sample. Second, the overall training load of youth handball players may not be sufficient to lead to a diversification in the functional adaptation according to playing positions. This may be underlined by the $8-9$ years of training experience of the present sample and the fact that youth players, especially at the very beginning of their handball career, mostly have low training frequencies of 1-2 training sessions/week versus 3-4 sessions/week at testing times and one game/week, which is possibly insufficient to lead to the appropriate functional adaptations. These may not solely be a result of the current training load but also the rather small past training load. Therefore, the dose-response relationship of handball-specific training and the subsequent overall training load may not be sufficient to lead to diverging neuromuscular adaptations or changes in the tendon or bony structures of youth handball players [24]. Third, the positional specialization may be lower in youth than in senior players with the former being used more often in different positions. More specifically, a high number of techniques like blocking, screening, or initiating body contact as a defender are executed with both arms in equal distri- bution, possibly neutralizing the effects of the unilateral technique executions while passing and throwing.

\section{Limitations}

There are a few limitations that need to be addressed. The YBT-UQ may not be sensitive enough to detect changes in shoulder stability in a cohort unfamiliar with the test and test setting. Consequently, further research is needed to confirm the present results for other upper quarter field tests (e. g., closed kinetic chain upper extremity stability test, seated medicine ball throw). The study population represents an unequal distribution in terms of playing positions and the ratio of male and female players. However, the a priori power analysis yielded that based on a sample of $\mathrm{N}=180 \mathrm{sig}$ nificant differences between playing positions would be detectable. Further, only the dominant playing position of the athletes was assessed with the consequence of some subjects playing this position for most of the time during training and games but sometimes also playing other positions.

\section{Conclusion}

The on-court physical demands of the different playing positions in youth handball players do not lead to significant differences in shoulder mobility and stability as assessed through the YBT-UQ. The overall training load of youth handball players may not be sufficient to lead to a diversification in the functional adaptation according to playing positions. Identifying performance requirements in reach directions and differences between playing positions may enable the development of position-specific training programs with the goal of improving shoulder mobility and stability in youth handball players.

\section{Acknowledgement}

We acknowledge support by the Open Access Publication Fund of the University of Duisburg-Essen. The funding body is independent of the design of the study and collection, analysis, and interpretation of data and in writing the manuscript. Open access funding enabled and organized by the project DEAL. 


\section{Conflict of Interest}

The authors declare that they have no conflict of interest.

\section{References}

[1] Sarmento H, Marcelino R, Anguera MT et al. Match analysis in football: A systematic review. J Sports Sci 2014; 32: 1831-1843

[2] Hoppe WM, Brochhagen ], Baumgart C et al. Differences in anthropometric characteristics and physical capacities between junior and adult top-level handball players. Asian J Sports Med 2017; 8: 1-10

[3] Achenbach L, Clément A, Hufsky L et al. The throwing shoulder in youth elite handball: soft-tissue adaptations but not humeral retrotorsion differ between the two sexes. Knee Surg Sports Traumatol Arthros 2019; 27: 3937-3943

[4] Wagner H, Buchecker M, von Duvillard SP et al. Kinematic comparison of team handball throwing with two different arm positions. Int J Sports Physiol Perform 2010; 5: 469-483

[5] Srhoj V, Marinovic M, Rogulj N. Position specific morphological characteristics of top-level male handball players. Coll Antropol 2002; 26: $219-227$

[6] Bauer ], Schedler S, Fischer $S$ et al. Relationship between Upper Quarter Y Balance Test performance and throwing proficiency in adolescent Olympic handball players. BMC Sports Sci Med Rehabilitation 2020; 12: 50

[7] Zapartidis I, Skoufas D, Vareltzis I et al. Factors influencing ball throwing velocity in young female handball players. Open Sports Med 2009; 3: 39-43

[8] Luig P, Krutsch W, Henke T et al. Contact - but not foul play - dominates injury mechanisms in men's professional handball: a video match analysis of 580 injuries. Br J Sports Med 2020; 54: 984-990

[9] Michalsik LB. On-Court physical demands and physiological aspects in elite team handball. In: Laver L, Landreau P, Seil R. et al., Eds. Handball Sports Medicine: Basic Science, Injury Management and Return to Sport. Berlin: Springer; 2018: 15-33

[10] Póvoas S, Ascensão A, Magalhães ] et al. Physiological demands of elite team handball with special reference to playing position. J Strength Cond Res 2014; 28: 430-442

[11] Michalsik LB, Madsen K, Aagaard P. Technical match characteristics and influence of body anthropometry on playing performance in male elite team handball. J Strength Cond Res 2015; 29: 416-428
[12] Borms D, Cools A. Upper-extremity functional performance tests: reference values for overhead athletes. Int J Sports Med 2018; 39: 433-441

[13] Gorman P, Butler R, Plisky P et al. Upper Quarter Y Balance Test: Reliability and performance comparison between genders in active adults. J Strength Cond Res 2012; 26: 3043-3048

[14] Bauer ], Panzer S, Muehlbauer T. Side differences of upper quarter y balance test performance in sub-elite young male and female handball players with different ages. BMC Sports Sci Med Rehabilitation 2021; 13: 141

[15] Wilson L, Leddington Wright S, Neza D. The assessment of arm dominance in water polo players using the Upper Quarter $Y$ Balance Test. In: British Association of Sport and Exercise Sciences Annual Conference University of Central Lancashire. Lancashire: Preston; 2013

[16] Faul F, Erdfelder E, Lang AG et al. G * Power 3: A flexible statistical power analysis program for the social, behavioral, and biomedical sciences. Behav Res Methods 2007; 39: 175-191

[17] Harriss DJ, Macsween A, Atkinson G. Ethical standards in sport and exercise science research: 2020 update. Int J Sports Med 2019; 40: 813-817

[18] Schwiertz G, Brueckner D, Schedler S et al. Reliability and minimal detectable change of the Upper Quarter Y Balance Test in healthy adolescents aged 12 to 17 years. Int J Sports Phys Ther 2019; 14: 927-934

[19] Patel RK, Choudhary R. Balance ability possessed by handball players pertaining to different playing positions. Int J Appl Res (Delhi) 2016; 2: 481-483

[20] Brumitt J, Patterson C, Dudley R et al. Comparison of lower quarter Y-Balance test scores for female collegiate volleyball players based on competition level, position, and starter status. Int J Sports Phys Ther 2019; 14: 415-423

[21] Ryu C, Park J, Kang M et al. Differences in lower quarter Y-balance test with player position and ankle injuries in professional baseball players. J Orthop Surg (Hong Kong) 2019; 27: 1-7

[22] Butler RJ, Myers HS, Black D et al. Bilateral differences in the upper quarter function of high school aged baseball and softball players. Int ] Sports Phys Ther 2014; 9: 518-524

[23] Borms D, Maenhout A, Cools AM. Upper quadrant field tests and isokinetic upper limb strength in overhead athletes. J Athl Train 2016; 51: 789-796

[24] Andersson S, Bahr R, Clarsen B et al. Preventing overuse shoulder injuries among throwing athletes: A cluster-randomised controlled trial in 660 elite handball players. Br J Sports Med 2016; 51: $1073-$ 1080 